\title{
Guest editorial: pre-leukemia/pre-lymphoma-what is old, what is new?
}

\author{
Shigeru Chiba ${ }^{1}$
}

Received: 25 September 2015 / Accepted: 29 September 2015 / Published online: 20 October 2015

(C) The Japanese Society of Hematology 2015

In 1949, a small number of patients with anemia and predisposition to acute myeloid leukemia (AML) were referred to as "pre-leukemic anemia [1]." These patients were to be diagnosed as myelodysplastic syndromes (MDS), based on the present concept. The term "pre-leukemia" since then has been used to imply MDS in most documents, while chronic phases of chronic myeloid leukemia (CML) and myeloproliferative neoplasms (MPN) have also been characterized as pre-leukemia in more limited contexts.

A change has been brought by the introduction of second-generation sequencing technology. In 2012, Jan, et al. reported the presence of clonal hematopoietic stem cells (HSCs), having mutations in selected genes in the bone marrow of de novo AML patients achieving a complete remission [2]. Additional evidence conveyed by Shlush, et al., who used a xenogeneic transplant model, further indicated that de novo AML often has a pre-leukemic phase without any clinical manifestations [3]. Shlush reviews this tale. He further discusses currently expanding concept interconnecting aging, clonal hematopoiesis, and preleukemia.
Kikushige et al. reached a similar concept in 2011, using a biological approach. They found that HSCs isolated from chronic lymphocytic leukemia (CLL) patients can engraft and develop a lymphoproliferative condition, and further, CLL-like disease in immunodeficient mice when transplanted [4]. The genetic basis of such HSCs was recently described by Damm et al. actualizing the presence of HSCs that are in a pre-leukemic state [5]. Kikushige summarizes this, also referring to some other lymphoid neoplasms.

Malignant lymphomas are cancers of lymphoid tissues, with only an occasional invasion to the bone marrow. Naturally, the origin of tumor cells has been postulated to be various kinds of mature lymphocytes. Nevertheless, the mutations found in the lymphoma tissues were also discovered in apparently normal bone marrow or variously differentiated blood cells, or in MDS cells in the same patients [6-8]. Particularly, independent groups reached this conclusion in peripheral $\mathrm{T}$ cell lymphoma cases. The clonal origin of some lymphomas may lie at the hematopoietic stem cell level. SakataYanagimoto will tell us the story of pre-lymphoma cells.
Shigeru Chiba

schiba-tky@umin.net

1 Department of Hematology, Faculty of Medicine, University of Tsukuba, Tsukuba, Japan 


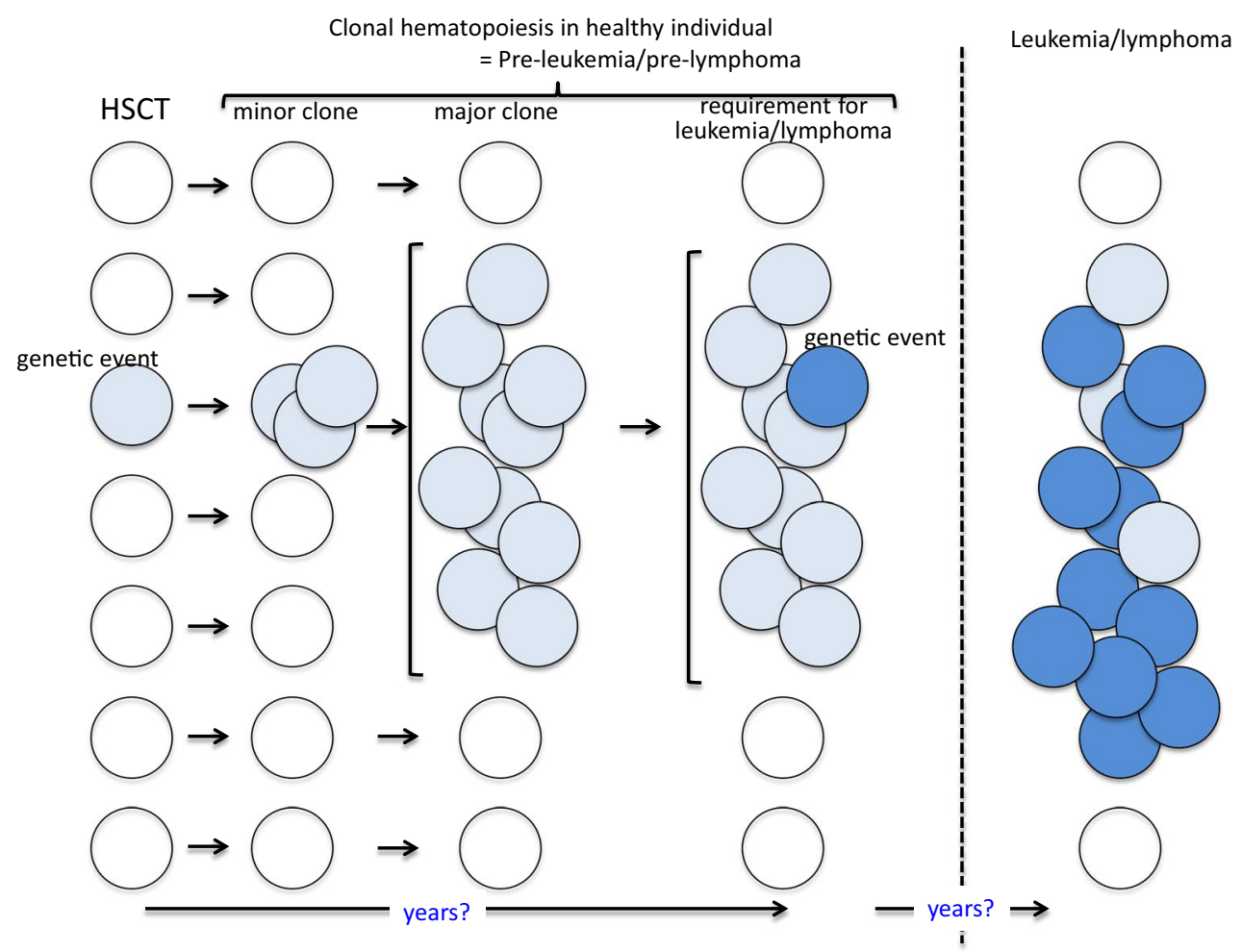

Fig. 1 Concept of pre-leukemia/pre-lymphoma

A concept of pre-leukemia/pre-lymphoma is illustrated in Fig. 1.

\section{References}

1. Hamilton-Paterso JL. Pre-leukaemic anaemia. Acta Haematol. 1949;2(5):309-16.

2. Jan $\mathrm{M}$, et al. Clonal evolution of preleukemic hematopoietic stem cells precedes human acute myeloid leukemia. Sci Transl Med. 2012;4(149):149ra118.

3. Shlush LI, et al. Identification of pre-leukaemic haematopoietic stem cells in acute leukaemia. Nature. 2014;506(7488):328-33.
4. Kikushige Y, et al. Self-renewing hematopoietic stem cell is the primary target in pathogenesis of human chronic lymphocytic leukemia. Cancer Cell. 2011;20(2):246-59.

5. Damm F, et al. Acquired initiating mutations in early hematopoietic cells of CLL patients. Cancer Discov. 2014;4(9):1088-101.

6. Quivoron C, et al. TET2 inactivation results in pleiotropic hematopoietic abnormalities in mouse and is a recurrent event during human lymphomagenesis. Cancer Cell. 2011;20(1):25-38.

7. Couronne L, et al. TET2 and DNMT3A mutations in human T-cell lymphoma. N Engl J Med. 2012;366(1):95-6.

8. Sakata-Yanagimoto M, et al. Somatic RHOA mutation in angioimmunoblastic T cell lymphoma. Nat Genet. 2014;46(2):171-5. 